\title{
Avaliação física de ovos comerciais de diferentes espécies de aves
}

\author{
Physical evaluation of commercial eggs of different species
}

Emanuel Neto Alves de Oliveira ${ }^{[a]}$, Joabis Nobre Martins ${ }^{[a]}$, Dyego da Costa Santos ${ }^{[a]}$

[a] Tecnólogos em Alimentos, mestrandos em Engenharia Agrícola pela Universidade Federal de Campina Grande (UFCG),

Campina Grande, PB - Brasil, e-mails: emanuelnetoliveira@ig.com.br; martins_ta@hotmail.com; dyego.csantos@gmail.com

\section{Resumo}

O objetivo do trabalho foi avaliar e comparar as características físicas de ovos comerciais. Os experimentos foram conduzidos com ovos brancos de galinha de granja (GB) e vermelhos (GV), galinha caipira (CP1 e CP2), pata (PT1 e PT2) e galinha da Angola (AG1 e AG2), avaliando-se as cascas, albúmens, gemas inteiras e homogeneizadas e mistura do albúmen e gema. Os parâmetros avaliados foram: massa individual, diâmetro transversal e longitudinal, coloração (luminosidade e intensidade de vermelho e amarelo) e textura (adesividade e compressão). Verificou-se que a massa individual variou de 38,08 (AG2) a 72,38 g (PT1). Os maiores diâmetros transversais foram encontrados nos tratamentos PT1 (45,50 mm) e GB (45,00 mm). Os ovos dos tratamentos GV, GB e CP1 apresentaram as maiores porcentagens de albúmen, e os tratamentos PT1 e PT2, as maiores porcentagens de gema. As maiores luminosidades foram verificadas nas cascas dos tratamentos GB, AG1, PT1 e PT2. Os valores de intensidade de amarelo variaram de 2,54 (casca do tratamento GB) a 99,57 (mix do tratamento CP1). Quanto à textura, os valores de adesividade variaram de -12,33 (albúmen do tratamento CP1) a -71,53 N (gema do tratamento PT2), e os de compressão variaram de 7,13 (albúmen do tratamento CP1) a 58,38 N (gema do tratamento PT1). Os tratamentos GV, GB e CP1 são recomendados para o processamento de produtos que necessitam de grandes quantidades de albúmen, enquanto que os tratamentos PT1 e PT2 são indicados para o processamento de produtos que necessitem sensorialmente de maior adesividade.

Palavras-chave: Controle de qualidade. Colorimetria. Textura.

\section{Abstract}

The objective of this study was to evaluate and compare the physical characteristics of commercial eggs. The experiments were conducted with white (GB) and red (GV) chicken eggs from layers raised in large-scale farms, home-produced chicken eggs (CP1 and CP2), paw eggs (PT1 and PT2) and chicken eggs of Angola layers (GN1 and GN2). The eggshell, albumen, yolk and mixture of albumen and yolk were evaluated. The parameters analyzed were: individual mass, transverse and longitudinal diameters, color (luminosity and intensities of red and yellow) 
and texture (adhesiveness and compression). The individual mass of the eggs examined varied from 38.08 (AG2) to $72.38 \mathrm{~g}$ (PT1). The largest transverse diameter was found for PT1 (45.50 mm), followed by GB (45.00 mm). GV, $G B$ and CP1 presented higher percentages of albumen content, while PT1 and PT2 showed higher percentages of yolk. Moreover, higher luminosity levels were found for shells belonging to GB, AG1, PT1 and PT2 eggs. The values of yellow intensity ranged from 2.54 (GB eggshell) to 99.57 (CP1 yolk + albumen mixture). In addition, the texture analyses showed adhesion values ranging from -12.33 (CP1 albumen) to -71.53 N (PT2 yolk) and compression values varying from 7.13 (CP1 albumen) to $58.38 \mathrm{~N}$ (PT1 yolk). The present results suggest the utilization of GV, $G B$ and CP1 eggs for the processing of products which require large quantities of albumen, whereas PT1 and PT2 eggs are indicated for the preparation of products requiring higher adhesion.

Keywords: Quality control. Colorimetry. Texture.

\section{Introdução}

Outrora, os ovos eram comercializados para aperitivos ou ornamentação de pratos. Atualmente, eles são comercializados sob inúmeras formas de apresentação, pois são considerados a fonte mais confiável de muitos compostos, como proteínas, lipídios, aminoácidos essenciais, vitaminas e minerais (BISCARO; CANNIATTI-BRAZACA, 2006; SEIBEL et al., 2010).

Segundo Bertechini (2003), o ovo é um recipiente biológico que contém material orgânico e inorgânico em sua constituição. Apresenta como componentes principais a gema, que representa cerca de 30\% do peso do ovo (OLIVEIRA et al., 2010), composta por lipídeos (BELITZ; GROSCH, 1997), o albúmen (ou clara) (cerca de 60\% do peso do ovo) (OLIVEIRA et al., 2010), do qual 88\% corresponde à água e $12 \%$ são proteínas (SALINAS, 2002). A casca representa cerca de $10 \%$ do peso do ovo fresco (OLIVEIRA et al., 2010), contendo $90 \%$ de minerais dentro de uma estrutura ou matriz orgânica, dos quais $98 \%$ correspondem ao cálcio na forma de cristais (BELITZ; GROSCH, 1997).

A qualidade do ovo pode ser analisada sob as óticas de mercado, produtor, consumidor e indústria processadora. No tocante ao mercado, a qualidade recebe diferentes considerações na dependência dos estágios de produção e comercialização. Para o produtor, a qualidade significa peso e resistência da casca, por outro lado, para os consumidores significa prazo de validade e boas características sensoriais. Por fim, para os processadores, indica facilidade de retirada da casca, cor da gema e propriedades funcionais (ALLEONI; ANTUNES, 2001).
Vários autores relatam que dentre os atributos sensoriais, a cor tem sido relacionada como indicador de qualidade, exercendo papel importante na aceitação dos alimentos pelos consumidores (SILVA et al., 2000; PEREIRA et al., 2001; TOCCHINI; MERCADANTE, 2001). Em ovos, a coloração da gema é a característica interna mais observada pelo consumidor, apesar de ser uma medida subjetiva, que varia do amarelo claro ao laranja avermelhado (OLIVEIRA et al., 2010). De acordo com Moura et al. (2009), a cor da gema é comumente relacionada à qualidade nutricional e, muitas vezes, é ferramenta decisória para a aquisição do produto pelo consumidor. Porém, o sabor é a característica sensorial que determina a preferência do consumidor pelo produto em questão.

Normalmente, uma coloração mais forte da gema em ovos de poedeiras comerciais é desejável e depende exclusivamente da alimentação fornecida, uma vez que esses animais não são capazes de sintetizar pigmentos de cor, mas podem absorver de $20 \%$ a $60 \%$ dos pigmentos da ração (STADELMAN; COTTERILL, 1995; LEE et al., 2001; SANTOS-BOCANEGRA et al., 2004). Segundo Garcia et al. (2002), a cor da gema dos ovos resulta da deposição de xantofilas (grupo de pigmentos carotenoides) nesse componente do ovo.

Segundo Hencken (1992), a deposição de pigmentos na gema dos ovos e a intensidade de sua coloração estão diretamente relacionadas ao consumo de alimentos que contenham pigmentos carotenoides em sua constituição pelas aves.

Vários autores já pesquisaram a coloração e o rendimento de componentes de ovos. Silva et al. (2000) estudaram o efeito do extrato de urucum na 
pigmentação da gema dos ovos; Silva et al. (2006) analisaram o resíduo da semente de urucum (Bixa Orellana L.) como corante da gema, pele, bico e ovário de poedeiras avaliado por dois métodos analíticos; Biscaro e Caniatti-Brazaca (2006) avaliaram a cor, o betacaroteno e o colesterol em gema de ovos obtidos de poedeiras que receberam diferentes dietas; Costa et al. (2008) investigaram a influência do óleo de linhaça sobre o desempenho e a qualidade dos ovos de poedeiras semipesadas; Garcia et al. (2009) avaliaram o desempenho e a qualidade dos ovos de poedeiras comerciais alimentadas com semente de urucum moída na dieta; Oliveira et al. (2010) estudaram as fontes de lipídios na dieta de poedeiras, o desempenho produtivo e a qualidade dos ovos.

No entanto, não foram encontrados na literatura consultada trabalhos relacionados à avaliação de textura em ovos in natura. Sabe-se que a textura é um dos atributos sensoriais de maior importância em alimentos e está diretamente relacionada com o seu estado físico. De acordo com Rodrigues (1999), nos alimentos em geral, a textura é um dos atributos mais importantes que afetam a preferência e a aceitação por parte dos consumidores. Para cada alimento, existe uma série de fatores básicos de qualidade e uma série de características de textura que são apreciados.

Diante do exposto, o objetivo do trabalho foi avaliar as características físicas de ovos comerciais de aves e de seus constituintes.

\section{Materiais e métodos}

Este estudo foi realizado com ovos de galinha de granja brancos (GB) e vermelhos (GV), galinha caipira (CP1 e CP2), pata (PT1 e PT2) e galinha da Angola (AG1 e AG2). Os ovos foram adquiridos no mercado varejista de Campina Grande (PB). Ao realizar a coleta das amostras, observou-se o modo de armazenamento, o estado de conservação, a qualidade externa dos ovos (presença de fezes e rachaduras na casca) e a integridade das embalagens, quando presente, com o objetivo de se obter amostragem homogênea.

Os ensaios foram realizados com 20 ovos de cada tratamento no Laboratório de Armazenamento e Processamento de Produtos Agrícolas (LAPPA), da Unidade Acadêmica de Engenharia Agrícola (UAEA), do Centro de Tecnologia e Recursos Naturais (CTRN) da Universidade Federal de Campina Grande (UFCG), na cidade de Campina Grande (PB). Após a recepção no Laboratório, os ovos foram submetidos à lavagem, com a finalidade de remover possíveis resíduos aderidos nas cascas. Nessa etapa foi utilizado detergente neutro de grau alimentício. Logo após, os ovos foram enxaguados em água corrente e secos com papel toalha.

Os ovos foram pesados individualmente em balança analítica (precisão 0,001 g). Em seguida, foram separados a casca, o albúmen e a gema e calculado o rendimento desses constituintes nos ovos de cada tratamento, de acordo com a seguinte equação:

$\mathrm{RD}=\frac{\mathrm{PC} \times 100}{\mathrm{P} \text { ? }}$

Em que:

RD: rendimento;

PC: massa do constituinte (gema ou albúmen);

POV: massa do ovo.

Os diâmetros longitudinal e transversal foram medidos com paquímetro digital. A análise de cor foi realizada na superfície da casca, nas gemas inteiras e homogeneizadas, no albúmen e no mix. Na composição do tratamento mix, o albúmen e a gema foram adicionados, em sua totalidade, em um béquer e homogeneizados. A coloração foi determinada utilizando-se o colorímetro MiniScan HunterLab XE Plus modelo 4500 L, com determinação dos seguintes parâmetros: $\mathrm{L}^{*}$ - luminosidade $\left(\mathrm{L}^{*}=0\right.$ - preto e $L^{*}=100$ - branco); $a^{*}$ - transição da cor verde $\left(-a^{*}\right)$ para o vermelho $\left(+a^{*}\right)$; e b* - transição da cor azul $\left(-b^{*}\right)$ para a cor amarela $\left(+b^{*}\right)$.

A adesividade e a compressão foram realizadas na gema homogeneizada, albúmen e mix, sendo determinadas em texturômetro universal (TA-XTPlus Texture Analyzer, da Stable Micro Systems), com probe cilíndrico (P/36R: $36 \mathrm{~mm}$ dia aluminium radiused AACC), com célula de carga de $50 \mathrm{~kg}$, equipado com o software Exponent Stable Micro Systems. As amostras foram padronizadas para análise, utilizando-se amostras de 1,0 mL dos componentes do ovo em superfície plana do equipamento para realização das medições. 0 experimento foi realizado em uma sala com temperatura ambiente controlada $\left(22^{\circ} \mathrm{C}\right)$. 
Os parâmetros utilizados para a realização das medições da textura foram os seguintes: velocidade pré-teste de 1,0 mm/s; velocidade de teste de 1,7 $\mathrm{mm} / \mathrm{s}$; velocidade pós-teste de $10 \mathrm{~mm} / \mathrm{s}$ e distância de $99 \%$. Os resultados obtidos foram expressos em Newton (N). Os valores dos picos máximos de adesividade e compressão foram registrados para cada componente do ovo na curva do software e a média das três medições, para cada componente, foi usada na análise estatística.

0 delineamento experimental das análises físicas dos ovos foi o de blocos inteiramente casualizados, com oito tratamentos e 20 repetições, utilizando-se o software Assistat (SILVA; AZEVEDO, 2009). Os dados foram submetidos à análise de variância (ANOVA) e a comparação de médias foi feita pelo teste de Tukey ao nível de 5\% de probabilidade.

\section{Resultados e discussão}

Na Tabela 1 estão apresentados os valores médios para massa individual, rendimento (albúmen e gema) e diâmetro dos ovos estudados.

Comparando-se os tratamentos entre si, observa-se que houve diferença significativa em nível de 1\% de probabilidade para todos os parâmetros analisados; no entanto, na comparação dos tratamentos de ovos da mesma espécie, verifica-se que os ovos de granja (GB e GV), caipira (CP1 e CP2) e pata (PT1 e PT2) não apresentaram diferença significativa entre si pelo teste de Tukey $(\mathrm{p} \leq 0,05)$ para todos os parâmetros estudados. Apenas os ovos de galinha da Angola (AG1 e AG2) apresentaram diferença significativa para massa individual e diâmetro longitudinal e transversal (Tabela 1).

De acordo com o apresentado na Tabela 1, a massa individual dos ovos variou entre 38,08 (AG2) e 72,38 g (PT1). Os valores encontrados para a massa individual estão próximos dos verificados por Garcia et al. (2009), que, ao estudarem o desempenho e a qualidade de ovos de poedeiras comerciais alimentadas com semente de urucum, obtiveram massa individual variando entre 53,52 g e 57,06 g, e Oliveira et al. (2010), que, ao estudarem a adição de fontes de lipídios na dieta de poedeiras, verificaram massa individual variando entre 53,45 g e 54,58 g. De acordo com Roberts (2004), o peso do ovo pode aumentar de acordo com a idade da ave. Como os ovos foram adquiridos no varejo, não se soube informar se a idade das aves foi um fator de influência nesses resultados.

Quanto aos diâmetros dos ovos (Tabela 1), os maiores diâmetros longitudinais foram revelados nos tratamento PT1 $(65,50 \mathrm{~mm})$ e PT2 $(61,83$ $\mathrm{mm})$, e os menores nos ovos AG2 (48,50 mm) e AG1 (55,33 mm). Os maiores diâmetros transversais foram encontrados nos tratamento PT1 $(45,50 \mathrm{~mm}) \mathrm{e}$ GB $(45,00 \mathrm{~mm})$ e os menores nos tratamentos AG2 (37,83 mm) e AG1 (41,00 mm). Observa-se uma relação direta entre os diâmetros e as massas individuais dos ovos, pois os tratamentos que apresentaram as maiores e as menores massas foram também os que apresentaram os maiores e os menores diâmetros, respectivamente.

No tocante ao rendimento (albúmen e gema), os tratamentos GV, GB e CP1 apresentaram as maiores porcentagens de albúmen (60,37\%; 59,11\% e $59,02 \%$, respectivamente), e os tratamentos PT1 e PT2, as maiores porcentagens de gema $(50,72 \%$ e $45,38 \%$, respectivamente).

Os valores experimentais para a porcentagem de albúmen foram inferiores aos verificados por Costa et al. (2008), que obtiveram variação de $64,29 \%$ a $65,27 \%$, e Oliveira et al. (2010), que observaram variação de $64,82 \%$ a $65,76 \%$ de rendimento para esse constituinte. Em contrapartida, os valores revelados para as porcentagens de rendimento da gema são superiores aos encontrados pelos mesmos autores (COSTA et al., 2008; OLIVEIRA et al., 2010), que verificaram porcentagem de rendimento de gema variando de $25,40 \%$ a $26,25 \%$ e de $25,18 \%$ a $26,13 \%$, respectivamente. Segundo Roberts (2004), a proporção de gema tende a ser menor em ovos pequenos.

No tocante à determinação de luminosidade $\left(\mathrm{L}^{*}\right)$, observa-se que as maiores intensidades foram verificadas nas cascas dos tratamentos GB $(90,63)$, AG1 $(81,71)$, PT1 $(81,41)$ e PT2 $(81,36)$. Os valores de luminosidade variaram de 54,44 na gema homogeneizada do tratamento AG2 a 90,63 na casca do tratamento GB (Tabela 2).

Observa-se que os valores encontrados na gema inteira foram superiores aos valores da gema homogeneizada, para todos os tratamentos. Os valores revelados para os ensaios de luminosidade da gema inteira e homogeneizada foram superiores aos encontrados por Garcia et al. (2009), que obtiveram variação de 49,68 a 53,03, semelhantes aos 
Tabela 1 - Resultados das análises físicas e rendimento dos ovos

\begin{tabular}{|c|c|c|c|c|c|}
\hline \multirow[b]{2}{*}{ Tratamento } & \multirow{2}{*}{$\begin{array}{c}\text { Massa } \\
\text { individual (g) }\end{array}$} & \multirow{2}{*}{$\begin{array}{l}\text { Rendimento do } \\
\text { albúmen (\%) }\end{array}$} & \multirow{2}{*}{$\begin{array}{l}\text { Rendimento da } \\
\text { gema (\%) }\end{array}$} & \multicolumn{2}{|c|}{ Diâmetro médio (mm) } \\
\hline & & & & Longitudinal (a) & Transversal (b) \\
\hline GB & $65,17^{b}$ & $59,11^{\mathrm{a}}$ & $28,30^{\mathrm{bc}}$ & $58,67^{\mathrm{bc}}$ & $45,00^{\mathrm{a}}$ \\
\hline GV & $65,39 \mathrm{~b}$ & $60,37^{a}$ & $25,85^{c}$ & $58,17^{\mathrm{bc}}$ & $44,83^{a}$ \\
\hline CP1 & $56,72^{c}$ & $59,02^{\mathrm{a}}$ & $27,64^{\mathrm{bc}}$ & $55,67^{c}$ & $42,83^{\mathrm{ab}}$ \\
\hline CP2 & $52,86^{\mathrm{cd}}$ & $53,34^{\mathrm{a}}$ & $31,34^{\mathrm{bc}}$ & $55,50^{c}$ & $41,50^{b}$ \\
\hline AG1 & $50,24^{d}$ & $51,58^{\mathrm{ab}}$ & $36,23^{b}$ & $55,33^{c}$ & $41,00^{b}$ \\
\hline AG2 & $38,08 \mathrm{e}$ & $42,61^{b c}$ & $35,62^{b}$ & $48,50^{d}$ & $37,83 \mathrm{c}$ \\
\hline PT1 & $72,38^{a}$ & $31,33^{d}$ & $50,72^{\mathrm{a}}$ & $65,50^{\mathrm{a}}$ & $45,50^{\mathrm{a}}$ \\
\hline PT2 & $71,46^{a}$ & $37,62^{\text {cd }}$ & $45,38^{a}$ & $61,83^{\mathrm{ab}}$ & $44,83^{a}$ \\
\hline MG & 59,04 & 49,37 & 35,13 & 57,40 & 42,92 \\
\hline DMS & 5,86 & 10,40 & 9,10 & 4,68 & 2,71 \\
\hline CV (\%) & 3,51 & 7,45 & 9,16 & 2,88 & 2,23 \\
\hline Fcal & $96,84^{* *}$ & $26,61^{* *}$ & $22,89^{* *}$ & $27,78^{* *}$ & $23,32^{* *}$ \\
\hline
\end{tabular}

Fonte: Dados da pesquisa

Legenda: $\mathrm{GB}=$ = Granja Branco; GM = Granja Vermelho; CP1 = Caipira tratamento 1; CP2 = Caipira tratamento 2; AG1 = Angola tratamento 1; AG2 = Angola tratamento 2; PT1 = Pata tratamento 1; PT2 = Pata tratamento 2; MG = Média geral; DMS = Desvio médio significativo; $C$ = Coeficiente de variação; Fcal = F calculado. Médias seguidas da mesma letra, na coluna, não diferem estaticamente a $5 \%$ de probabilidade, pelo teste de Tukey; $n$ s = não significativo, ${ }^{*}=$ significativo a $5 \% ;{ }^{* *}=$ significativo a $1 \%$ de probabilidade, pelo teste $\mathrm{F}$.

de Harder et al. (2007), que encontraram variação de 57,48 a 63,23, e inferiores aos de Biscaro e Canniatti-Brazaca (2006), que observaram variação entre 64,50 e 68,95 para luminosidade em gemas de ovos de poedeiras.

Comparando-se a luminosidade entre os tratamentos, observa-se diferença $(p<0,01)$. Analisando-se os tratamentos individualmente, verifica-se que os ovos CP1 e CP2 (albúmen), GB e GV (gema inteira e homogeneizada), PT1 e PT2 (gema homogeneizada, mix e casca), AG1 e AG2 (mix), não diferiram estaticamente pelo teste de Tukey ( $\mathrm{p} \leq 0,05)$.

No tocante à intensidade da cor transição verde-vermelho ( $a^{*}$ ), observa-se variação de -3,52 (albúmen do tratamento GV) a 31,20 (gema inteira do tratamento AG2). Em todos os ovos estudados, as maiores intensidades de vermelho foram reveladas nas gemas inteiras e homogeneizadas. Os resultados encontrados para cromaticidade $\left(a^{*}\right)$ das gemas foram superiores aos verificados por Biscaro e Canniatti-Brazaca (2006), que obtiveram variação entre -2,40 e 4,10, Harder et al. (2007), que obtiveram variação de 2,23 a 9,61, e Pereira (2009), que encontrou variação de -4,42 a -3,54 ao estudar o efeito dos lipídeos da ração sobre a qualidade, composição e estabilidade dos ovos de poedeiras comerciais.

Segundo Freitas et al. (2011), a pigmentação da gema pode variar de amarelo levemente claro a laranja escuro, de acordo com a alimentação e características individuais da ave. No entanto, como os ovos do presente estudo eram provenientes do comércio local, não se pode afirmar o nível de contribuição e a influência da alimentação das aves nos resultados.

De modo geral, verificou-se tendência à cor verde para o parâmetro a* (transição verde-vermelho) nos albúmens de todos os tratamentos, visto que os valores foram negativos. Observou-se, ainda, diferença significativa $(\mathrm{p}<0,01)$ para a cromaticidade $\left(a^{*}\right)$ em todos os tratamentos (casca, albúmen, gemas e mix) avaliados. Comparando-se os tratamentos em si dentro da mesma espécie animal, os ovos GP1 e GP2 (albúmen, gema inteira, gema homogeneizada e casca), AG1 e AG2 (albúmen e mix), GB e GV (gema homogeneizada) e PT1 e PT2 (gema homogeneizada, mix e casca) não diferiram estaticamente pelo teste de Tukey. 


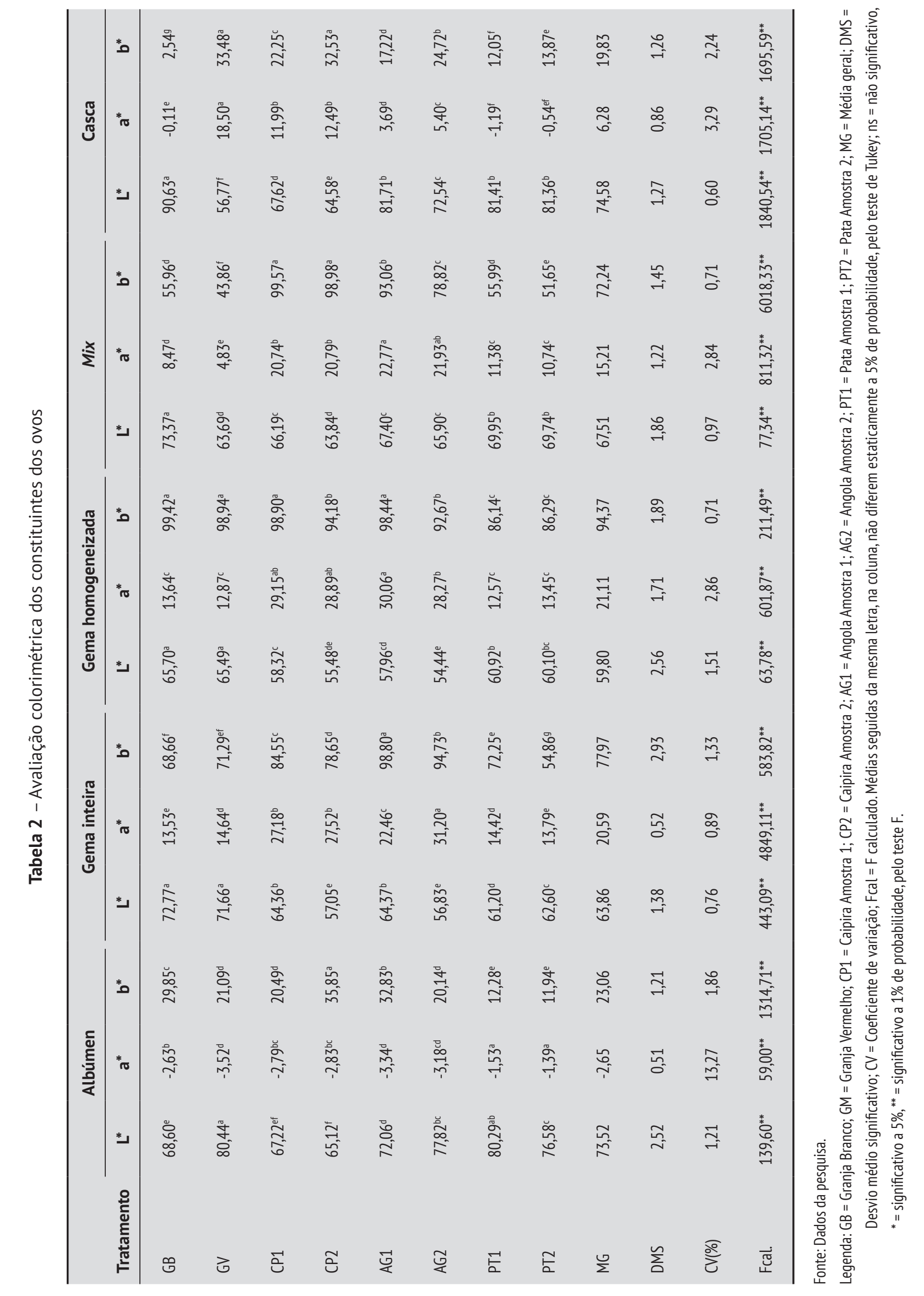

Rev. Acad., Ciênc. Agrár. Ambient., Curitiba, v. 9, n. 4, p. 415-423, out./dez. 2011 
Os valores revelados para a intensidade de amarelo $\left(b^{*}\right.$ ) variaram de 2,54 (casca do tratamento GB) a 99,57 (mix do tratamento GP1). Observa-se que os valores da gema inteira foram inferiores aos da gema homogeneizada, com exceção dos tratamentos AG1 e AG2.

Os valores encontrados para a intensidade de amarelo $\left(b^{*}\right)$ da gema inteira e homogeneizada foram superiores aos de Garcia et al. (2009), ao verificarem variação de 18,38 a 35,72, Harder et al. (2007), que encontraram variação de 49,15 a 53,42; Pereira (2009), que observou variação de 34,75 a 43,92 e semelhantes aos encontrados por Biscaro e Canniatti-Brazaca (2006), que obtiveram variação entre 52,43 a 55,18.

Paz et al. (2010), citando alguns autores, relatam que a cor da gema de ovos, principalmente o valor de b*, deve ser maior que 47 para que sua aceitação pelo consumidor não seja prejudicada. No presente estudo, o valor verificado para as gemas inteira e homogeneizada de todos os tratamentos foi superior ao recomendado para uma boa aceitação.

Na Tabela 3 estão apresentados os resultados de adesividade e compressão nos diferentes tratamentos estudados. Os valores de adesividade variaram de -12,33 (albúmen do tratamento CP1) a -71,53 N (gema do tratamento PT2). Em todos os tratamentos estudados, os albúmens apresentaram a menor adesividade, e a gema, a maior adesividade. Comparando-se os tratamentos individualmente, observa-se diferença estatística ( $\mathrm{p} \leq 0,05)$ entre todos os constituintes avaliados. A adesividade é representada nos gráficos pelos picos na porção negativa de força ao final de cada teste, onde o aumento da energia para remoção do probe das amostras durante a análise de textura foi evidenciado pelo aumento da adesividade.

No tocante à compressão, verifica-se variação de 7,13 (albúmen do tratamento CP1) a 58,38 N (gema do tratamento PT1). De forma semelhante à adesividade, as maiores compressões foram verificadas nas gemas e as menores nos albúmens para todos os tratamentos estudados, onde os albúmens, as gemas e o mix apresentaram diferença estatística ( $p$ $\leq 0,05 \%)$.

A adesividade e a compressão são dois parâmetros físicos relacionados à textura que estão diretamente relacionados ao processamento dos ovos,

Tabela 3 - Valores médios das análises de textura do albúmen, gema e mix

\begin{tabular}{|c|c|c|c|c|c|c|}
\hline \multirow[b]{2}{*}{ Tratamento } & \multicolumn{3}{|c|}{ Adesividade (N) } & \multicolumn{3}{|c|}{ Compressão (N) } \\
\hline & Albúmen & Gema & Mix & Albúmen & Gema & Mix \\
\hline GB & $-14,99^{\mathrm{bA}}$ & $-69,15^{a c}$ & $-31,45^{a B}$ & $8,46^{\mathrm{cdC}}$ & $45,58^{\mathrm{dA}}$ & $15,15^{\mathrm{dB}}$ \\
\hline GV & $-17,13^{\text {cA }}$ & $-70,41^{\mathrm{bcc}}$ & $-30,93^{\mathrm{a}}$ & $8,92^{c C}$ & $47,27^{\mathrm{dA}}$ & $14,86^{\mathrm{dB}}$ \\
\hline CP1 & $-12,33^{\mathrm{aA}}$ & $-69,50^{\mathrm{abc}}$ & $-32,68^{a B}$ & $7,13^{\mathrm{ec}}$ & $50,24^{\text {bca }}$ & $15,34^{\mathrm{cdB}}$ \\
\hline $\mathrm{CP} 2$ & $-14,19^{b A}$ & $-69,26^{a b c}$ & $-33,93^{a b B}$ & $7,83^{\text {dec }}$ & $51,91^{\mathrm{bA}}$ & $17,19^{с в}$ \\
\hline AG1 & $-17,41^{\mathrm{cA}}$ & $-69,19 \mathrm{abc}$ & $-37,57^{\mathrm{c} c \mathrm{~B}}$ & $10,32^{\mathrm{bc}}$ & $47,63^{\mathrm{cdA}}$ & $16,63^{\mathrm{cdB}}$ \\
\hline AG2 & $-13,44^{\mathrm{abA}}$ & $-71,48^{c c}$ & $-38,88^{\mathrm{CB}}$ & $10,12^{b c}$ & $51,15^{\mathrm{bA}}$ & $20,61^{\mathrm{bB}}$ \\
\hline PT1 & $-22,01^{\mathrm{dA}}$ & $-70,98^{c c}$ & $-50,01^{\mathrm{eB}}$ & $12,77^{a c}$ & $58,38^{\mathrm{aA}}$ & $29,44^{\mathrm{aB}}$ \\
\hline PT2 & $-21,28^{d A}$ & $-71,53^{c c}$ & $-43,61^{\mathrm{dB}}$ & $12,19^{\mathrm{ac}}$ & $52,87^{\mathrm{DA}}$ & $20,83^{\mathrm{bB}}$ \\
\hline MG & $-16,60$ & $-70,19$ & $-37,38$ & 9,72 & 50,63 & 18,76 \\
\hline DMS & 1,63 & 1,23 & 4,15 & 0,93 & 2,73 & 1,85 \\
\hline $\mathrm{CV}(\%)$ & 8,99 & 15,48 & 9,39 & 3,39 & 1,91 & 3,48 \\
\hline Fcal. & $114,66^{* *}$ & $17,07^{* *}$ & $62,05^{* *}$ & $112,29^{* *}$ & $51,85^{* *}$ & $169,81^{* *}$ \\
\hline
\end{tabular}

Fonte: Dados da pesquisa.

Legenda: $G B=$ Granja Branco; $G V=$ Granja Vermelho; $C P 1=$ Caipira tratamento 1; $C P 2$ = Caipira tratamento 2; $A G 1$ = Angola tratamento 1; $A G 2=$ Angola tratamento 2; PT1 = Pata tratamento 1; PT2 = Pata tratamento 2; MG = Média geral; DMS = Desvio médio significativo; CV = Coeficiente de variação; Fcal = F calculado. Médias seguidas da mesma letra minúscula na coluna e maiúscula na linha não diferem entre si ao nível de $5 \%$ de probabilidade pelo teste de Tukey; ns = não significativo, ${ }^{*}=$ significativo a $5 \%,{ }^{* *}=$ significativo a $1 \%$ de probabilidade, pelo teste $\mathrm{F}$. 
principalmente quando eles são utilizados na elaboração de produtos em que é necessária a agitação da gema e do albúmen para obtenção de mistura homogênea com características sensoriais desejáveis, como no caso da redução do odor característico do ovo em bolos e tortas, que também apresentam maior maciez quando se utilizam ovos bem homogeneizados para sua elaboração.

\section{Conclusão}

Os tratamentos GV, GB e CP1 são recomendados ao processamento de produtos que necessitam de grandes quantidades de albúmen, visto que apresentaram os maiores valores de rendimento para esse constituinte do ovo. 0 mesmo pode-se dizer para os tratamentos PT1 e PT2, que apresentaram os maiores rendimentos de gema.

Os tratamentos PT1 e PT2 são indicados para o processamento de produtos que necessitem sensorialmente de maior adesividade, como a maionese, pois apresentaram as maiores forças adesivas tanto para o albúmen como para a gema e o mix.

Houve diferença significativa em nível de $1 \%$ de probabilidade entre todas as características físicas dos tratamentos dos ovos estudados e seus constituintes.

\section{Referências}

ALLEONI, A. C. C.; ANTUNES, A. J. Unidade Haugh como medida de qualidade de ovos de galinha armazenados sob refrigeração. Scientia Agrícola, v. 58, n. 4, p. 681-685, 2001.

BELITZ, H. D.; GROSCH, W. Química de los alimentos. Zaragoza: Acribia, 1997.

BERTECHINI, A. G. Ovo é saúde. Revista Avicultura Industrial, v. 94, n. 6, p. 40-42, 2003.

BISCARO, L. M.; CANIATTI-BRAZACA, S. G. Cor, betacaroteno e colesterol em gema de ovos obtidos de poedeiras que receberam diferentes dietas. Ciência e Agrotecnologia, v. 30, n. 6, p. 1130-1134, 2006.

COSTA, F. G. P. et al. Influência do óleo de linhaça sobre o desempenho e a qualidade dos ovos de poedeiras semipesadas. Revista Brasileira de Zootecnia, v. 37, n. 5, p. 861-868, 2008.
FREITAS, L. W. et al. Aspectos qualitativos de ovos comerciais submetidos a diferentes condições de armazenamento. Revista Agrarian, v. 4, n. 11, p. 66-72, 2011.

GARCIA, E. A. et al. Efeitos dos níveis de cantaxantina na dieta sobre o desempenho e qualidade de ovos de poedeiras comerciais. Revista Brasileira de Ciência e Tecnologia Avícola, v. 4, n. 1, p. 55-61, 2002.

GARCIA, E. A. et al. Desempenho e qualidade dos ovos de poedeiras comerciais alimentadas com semente de urucum (Bixa orellana L.) moída na dieta. Revista Veterinária e Zootecnia, v. 16, n. 4, p. 689-697, 2009.

HARDER, M. N. C. et al. Avaliação quantitativa por colorímetro digital da cor do ovo de galinhas poedeiras alimentadas com urucum (Bixa orellana). Revista Portuguesa de Ciências Agrárias, v. 10, n. 2, p. 339-342, 2007.

HENCKEN, H. Chemical and physiological behavior of feed carotenoids and their effects on pigmentation. Poultry Science, v. 71, n. 4, p. 711-717, 1992.

LEE, B. D. et al. Nutritive and economic values of high oil corn in layer diet. Poultry Science, v. 80, n. 11, p. 15271534, 2001.

MOURA, A. M. A. et al. Características sensoriais de ovos de codornas japonesas (Coturnix japonica Temminck e Schlegel, 1849) suplementadas com pigmentantes sintéticos e selenometionina. Ciência e Agrotecnologia, v. 33, n. 6, p. 1594-1600, 2009.

OLIVEIRA, D. D. et al. Fontes de lipídios na dieta de poedeiras: desempenho produtivo e qualidade dos ovos. Arquivo Brasileiro de Medicina Veterinária e Zootecnia, v. 62, n. 3, p. 718-724, 2010.

PAZ, I. C. L. A. et al. Qualidade e produção de ovos de poedeiras vermelhas alimentadas com diferentes níveis de farinha de carne e suplementadas com manjericão. Revista Agrarian, v. 3, n. 7, p. 71-77, 2010.

PEREIRA, A. V. et al. Bixina como agente pigmentante das gemas de ovos de poedeiras comerciais. In: REUNIÃO ANUAL DA SOCIEDADE BRASILEIRA DE ZOOTECNIA, 38., 2001, Piracicaba. Anais... Piracicaba: SBZ, 2001. p. 839-840.

PEREIRA, A. L. F. Efeito dos lipídeos da ração sobre a qualidade, composição e estabilidade dos ovos de poedeiras comerciais. 2009. 87 f. Dissertação (Mestrado em Ciência e Tecnologia de Alimentos) - Universidade Federal do Ceará, Fortaleza, 2009. 
ROBERTS, J. R. Factors affecting egg internal quality and egg shell quality in laying hens. Journal of Poultry Science, v. 41, n. 3, p. 161-177, 2004.

RODRIGUES, A. C. C. Influência dos aditivos na obtenção de mamão desidratado osmoticamente. 1999. 98 f. Dissertação (Mestrado em Engenharia de Alimentos) Universidade Estadual de Campinas, Campinas, 1999.

SALINAS, R. D. Alimentos e nutrição: introdução à Bromatologia. 3. ed. Porto Alegre: Artmed, 2002.

SANTOS-BOCANEGRA, E. et al. Evaluation of xanthophylls from Targetes erectus (Marigold Flower) and Capsicum sp. (Red Pepper Paprika) as a pigment for egg yolks compare with synthetic pigments. International Journal of Poultry Science, v. 3, n. 11, p. 685-689, 2004.

SEIBEL, N. F. et al. Caracterização sensorial de ovos de codornas alimentadas com dietas modificadas. Ciência e Tecnologia de Alimentos, v. 30, n. 4, p. 884-889, 2010.

SILVA, J. H. V. et al. Efeito do extrato de urucum na pigmentação da gema dos ovos. Revista Brasileira de Zootecnia, v. 29, n. 5, p. 1435-1439, 2000.
SILVA, J. H. V. et al. Resíduo da semente de urucum (Bixa Orellana L.) como corante da gema, pele, bico e ovário de poedeiras avaliado por dois métodos analíticos. Ciência e Agrotecnologia, v. 30, n. 5, p. 988-994, 2006.

SILVA, F. A. S. E.; AZEVEDO, C. A. V. Principal components analysis in the software assistat-statistical attendance. In: WORLD CONGRESS ON COMPUTERS IN AGRICULTURE, 7., 2009, Reno-NV-USA. Anais... Reno-NV-USA: American Society of Agricultural and Biological Engineers, 2009.

STADELMAN, W. J.; COTTERILL, O. J. Egg science and technology. 4. ed. New York: Haworth Food Products, 1995.

TOCCHINI, L.; MERCADANTE, A. Z. Extração e determinação, por CLAE, de bixina e norbixina em coloríficos. Ciência e Tecnologia de Alimentos, v. 21, n. 3, p. 310313, 2001.
Recebido: 30/10/2011

Received: 10/30/2011

Aprovado: $14 / 03 / 2012$

Approved: 03/14/2012 